\title{
Hyperthermal Atomic Oxygen Beam Irradiation Effect on the Hydrogenated Si-doped DLC Film
}

\author{
Kengo Kidena ${ }^{1}$, Minami Endo ${ }^{1}$, Hiroki Takamatsu ${ }^{1}$, Ryo Imai ${ }^{1}$, Masahito Niibe ${ }^{1}$, \\ Kumiko Yokota $^{2}$, Masahito Tagawa ${ }^{2}$, Yuichi Furuyama ${ }^{3}$, \\ Keiji Komatsu ${ }^{4}$, Hidetoshi Saitoh ${ }^{4}$ and Kazuhiro Kanda ${ }^{1, *}$ \\ 1 Laboratory of Advanced Science and Technology for Industry, University of Hyogo \\ 2 Faculty of Engineering, Kobe University \\ 3 Faculty of Maritime Sciences, Kobe University \\ 4 Department of Materials Science and Technology, Nagaoka University of Technology \\ *Corresponding author: Fax +81-791-58-0242, and/or e-mail: kanda@lasti.u-hyogo.ac.jp
}

\begin{abstract}
We investigated the effect of hyperthermal atomic oxygen beam irradiation on hydrogenated Si-doped Diamond-Like Carbon (hydrogenated Si-DLC) films for the purpose of use as a solid lubrication material in space. We found that film thickness of hydrogenated Si-DLC was constant after the exposure to atomic oxygen beam. From this result, the hydrogenated Si-DLC films have resistance to etching by the irradiation of atomic oxygen, unlike hydrogenated non-dope DLC films. In addition bulk composition of hydrogenated Si-DLC film kept constant. Especially, hydrogen content in Si-DLC film did not decrease. Therefore, hydrogenated Si-DLC film is expected to keep low fiction properties in a vacuum. Furthermore, the atomic oxygen beam fluence dependence of X-ray Photoelectron Spectroscopy (XPS) and Near Edge X-Ray Absorption Fine Structure (NEXAFS) spectra of hydrogenated Si-DLC films were measured. From these studies, it was found that the $\mathrm{C}$ atoms on the hydrogenated Si-DLC surface were desorbed, but $\mathrm{Si}$ atoms were remained on the hydrogenated Si-DLC surface as the $\mathrm{SiO}_{\mathrm{x}}$ by the collision of atomic oxygen. This $\mathrm{SiO}_{\mathrm{x}}$ layer was considerable to disturb the erosion of bulk film against atomic oxygen. From the element distribution in depth direction, it was found that the thickness of $\mathrm{SiO}_{\mathrm{x}}$ was about $5 \mathrm{~nm}$.

Key words: DLC, Si-doped DLC, NEXAFS, atomic oxygen, LEO,
\end{abstract}

\section{Introduction}

In the space, solid lubrication materials of substitution are required, because oil cannot be used due to it evaporates. In order to use as lubrication material in space, its resistance to extreme space environment, such as temperature, ultraviolet light, radiation of atomic oxygen, and their synergistic effects, becomes important. Artificial satellites are positioned the region called low-Earth orbit (LEO), where region less than $2000 \mathrm{~km}$ above the ground. Hyperthermal atomic oxygen is the dominant species and main cause of deterioration in LEO, and its the translation energy of $5 \mathrm{eV}^{1-4)}$. Therefore, for use in LEO it is necessary to confirm its resistance against the atomic oxygen irradiation.

Diamond-like carbon (DLC) films have been used tribological field as coating material on edged-tools, computer hard disks, automobile component and so on, because they have a several superior properties including low friction coefficient, high hardness, high wear and corrosion resistance ${ }^{5-10)}$. The characteristic of DLC film are correlated the $s p^{2} /\left(s p^{2}+s p^{3}\right)$ ratio of carbon and content of hydrogen. Hydrogenated DLC films are expected to be used as lubrication materials in LEO, because it was known that the DLC films with a hydrogen content greater than 40 at.\% provide ultra-low friction (friction coefficient less than 0.001 ) even under air and vacuum conditions ${ }^{11)}$. However, it was found that the hydrogenated DLC films were etched by the collision with hyperthermal atomic oxygen in our previous work 12). Therefore, the hydrogenated DLC films cannot be just used as a solid lubrication material in space.

In this study, we investigated the effect of hyperthermal atomic oxygen beam irradiation on hydrogenated Si-doped Diamond-Like Carbon (hydrogenated Si-DLC) film. In last decade, many working groups were reported the several properties of DLC films were improved by doping a hetero element ${ }^{13-19)}$. Especially, Si-doped DLC films have been widely investigated because it is expected improve sliding properties, burning resistance, and oxidation resistance 19-24). We performed atomic oxygen exposure to hydrogenated Si-DLC films with a collisional energy of 5 eV by using a laser detonation-type beam apparatus. Film thickness and bulk composition of hydrogenated Si-DLC were estimated by combination of Rutherford Backscattering Spectrometry (RBS) and Elastic Recoil Detection Analysis (ERDA). In addition, we performed the chemical analysis on the DLC film surface by 
measuring X-ray Photoelectron Spectroscopy (XPS) and Near Edge X-Ray Absorption Fine Structure (NEXAFS). Element distribution in depth direction of hydrogenated Si-DLC films was obtained from the measurement of the Glow Discharge Optical Emission Spectroscopy (GD-OES)

\section{Experimental methods}

The hydrogenated Si-DLC films used in this study are commercially available (Nippon ITF), which hydrogen content greater than 40 at. $\%$. The films were deposited at $1 \mu \mathrm{m}$ thickness on $\mathrm{Si}$ wafers using amplitude modulated RF plasma-enhanced chemical vapor deposition (CVD) ${ }^{25}$. The exposure of an hyperthermal atomic oxygen into the hydrogenated Si-DLC films was done using a laser detonation-type beam apparatus ${ }^{12.26 .27)}$. Pure oxygen gas is introduced into the nozzle throat through a pulsed supersonic valve. A pulse from a $\mathrm{CO}_{2}$ laser $(10.6 \mu \mathrm{m},>5$ $\mathrm{J} /$ pulse) is focused into the oxygen gas at the nozzle throat. By absorbing the laser energy, high-density and high-temperature oxygen plasma is formed at the nozzle throat. Once the plasma is formed, it propagated and absorbs the energy in the tail of the laser pulse. The plasma propagation occurs along at the incident laser axis, and oxygen molecules are decomposed and accelerated at the shock front of the plasma propagation. The typical atomic oxygen flux at the sample position $(46 \mathrm{~cm}$ from the nozzle) was estimated to be $3.51 \times 10^{15}$ atoms $\cdot \mathrm{cm}^{-2} \mathrm{~s}^{-1}$ by using a Ag-coated quartz crystal microbalance (QCM). The translational energy of hyperthermal atomic oxygen was estimated to be $5.46 \mathrm{eV}$ by using a time-of-flight (TOF) measurement system consisting of a quadrupole mass spectrometer with a scintillation detector and a multichannel scalar. This energy correspond the atomic oxygen energy in LEO. In the present study, four sheets of hydrogenated Si-DLC film were exposed to a hyperthermal atomic oxygen beam corresponding to the fluences of a) 0 atoms $\cdot \mathrm{cm}^{-2}$, b) $3.6 \times 10^{17}$ atoms $\cdot \mathrm{cm}^{-2}$, c) $1.2 \times 10^{18}$ atoms $\cdot \mathrm{cm}^{-2}$, d) $1.2 \times 10^{19}$ atoms $\cdot \mathrm{cm}^{-2}$.

Film thickness and bulk composition of hydrogenated Si-DLC film was estimated by the use of measuring the combination of ERDA and RBS. These measurement was performed using a tandem electrostatic accelerator (5SDH) located in the Faculty of Maritime Sciences, Kobe University ${ }^{12.28)}$. The sample was irradiated by 4.2 $\mathrm{MeV} \mathrm{He}^{2+}$ to accelerate the negative ion of helium $\left({ }^{4} \mathrm{He}\right)$ generated by the RF discharge negative- charge exchange ion source. Incident angle is $15^{\circ}$ with respect to the surface of the sample. In RBS, high-energy $\mathrm{He}^{2+}$ ions scattered elastically by the sample were captured with a solid-state detector (SSD) positioned at $160^{\circ}$ with respect to the beam direction.

Elementary composition of the hydrogenated Si-DLC film surface was estimated by the measuring the XPS, which was carried out using a conventional photoelectron spectroscopy apparatus (Shimazu ESCA-1000) mounted with a CL150 (VSW Ltd.) hemispherical electron energy analyzer. The $\mathrm{Mg} K \alpha$ line $(1253.6 \mathrm{eV})$ used as the X-ray source, was incident at $45^{\circ}$ with respect to the surface normal. The emission angle is $90^{\circ}$ with respect to the incident angle.

The NEXAFS measurements were carried out using total electron yield method at the NewSUBARU synchrotron radiation facility, which has a $1.5-\mathrm{GeV}$ electron storage ring ${ }^{29-32)}$. The beamline BL09A is to carry out surface analysis for various industrial materials with the use of high brilliant light source of the undulator and high resolution monochromator. The NEXAFS measurement of the $\mathrm{Si} K$ absorption was carried at BL05A, which have a bending magnet as light source. The C $K$ edge, Si $L$ edge, and $\mathrm{O} K$ edge measured at BL09A, which has a $11 \mathrm{~m}$-undulater as light source and grating monochromator and have a double crystal monochromator. In the measurement at both beamlines, irradiation angle irradiation angle to the sample is the "magic angle" of $54.7^{\circ}$ with respect to the surface. The NEXAFS spectra of the C $K$ edge absorption Si $L$ edge absorption, Si $K$ edge absorption, and $\mathrm{O} K$ edge absorption were measured in the range $275-300 \mathrm{eV}$, $100-108 \mathrm{eV}, 1820-1880 \mathrm{eV}$, and $520-560 \mathrm{eV}$, respectively. Element distribution in depth direction of hydrogenated Si-DLC films due to the irradiation of an atomic oxygen was estimated by the measurement of the Glow Discharge Optical Emission Spectroscopy (GD-OES). This measurement was performed using a HORIBA GD-Profiler2. The sample was sputtered by Ar plasma. The calculate quantitative chemical depth profiles can measure by causing atomic emission of sputtering atoms.

\section{Results and Discussion}

Variations in the film thickness and bulk composition of the hydrogenated Si-DLC films due to the irradiation of an atomic oxygen beam were analyzed from combination of RBS and ERDA. Fig. 1 shows the atomic oxygen beam fluence dependence of film thickness determined from RBS of hydrogenated Si-DLC films. The point of squares is shown film thickness of hydrogenated Si-DLC. The film thickness of hydrogenated non-doped DLC is also shown by point of circle for reference ${ }^{12}$. Film thickness of hydrogenated non-doped DLC film was reported to decrease with atomic oxygen fluence. On the other hand, that of hydrogenated Si-DLC film thickness was constant at about $1 \mu \mathrm{m}$ even after the exposure to atomic oxygen. In this result, hydrogenated Si-DLC films have resistance for etching due to exposure to atomic oxygen. Fig. 2 shows the atomic oxygen beam fluence dependence of measured intensity ratio of hydrogen were determined from combination of RBS and ERDA at hydrogenated Si-DLC films. Hydrogen ratio did not decrease after the exposure to atomic oxygen. Therefore, we found that hydrogen do not desorb in hydrogenated Si-DLC films by atomic oxygen irradiation. From the above RBS and ERDA results, hydrogenated Si-DLC films have resistance against etching by exposure to atomic oxygen. In addition, it is considered that the hydrogen content after irradiation is $40 \%$ or more. After exposure to atomic oxygen hydrogenated Si-DLC film, is expected to exhibit low friction properties in a vacuum. In this result, hydrogenated Si-doped DLC films are expected to be useful as a lubrication material in LEO. 


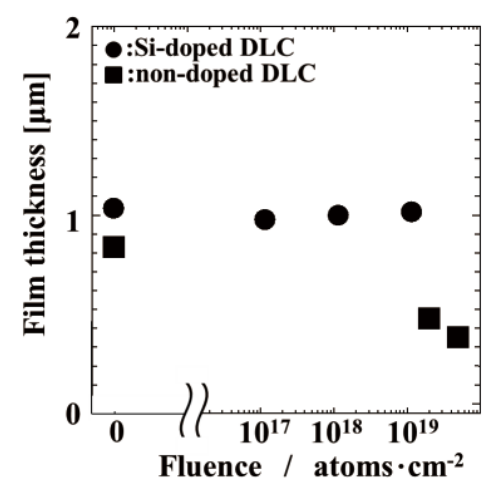

Fig. 1 Fluence dependence of film thickness was determined from RBS of hydrogenated Si-DLC films and hydrogenated non-doped DLC films. The point of circle and square is shown film thickness of hydrogenated Si-DLC film and hydrogenated non-doped DLC film, respectively.

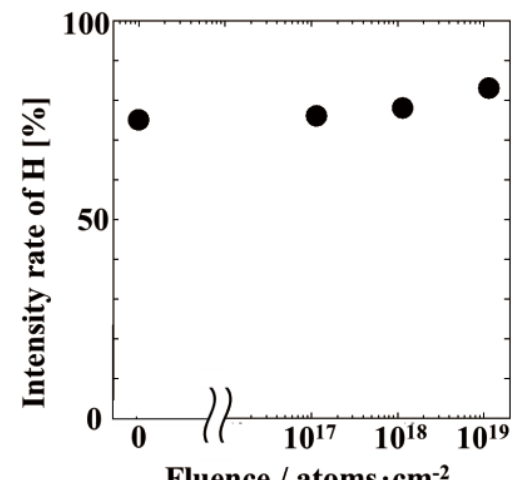

Fig. 2 Fluence dependence of measured intensity ratio of hydrogen was determined from combination of RBS and ERDA at hydrogenated Si-DLC films.

Next, we investigated the reason and mechanism for resistance of hydrogenated Si-DLC films to atomic oxygen irradiation. Elementary compositions of the surface of hydrogenated Si-DLC films were estimated by the measurement of the XPS spectra. Fig 3 shows that the flucence dependence of the $\mathrm{C} 1 s$ spectra measured by XPS of hydrogenated Si-DLC films. Atomic oxygen beam fluences are a) 0 atoms $\cdot \mathrm{cm}^{-2}$, b) $3.6 \times 10^{17}$ atoms . $\mathrm{cm}^{-2}$, c) $1.2 \times 10^{18}$ atoms $\cdot \mathrm{cm}^{-2}$, d) $1.2 \times 10^{19}$ atoms $\cdot \mathrm{cm}^{-2}$ in each figures. As shown in Fig. 3, intensity of the peak at $287 \mathrm{eV}$ decreased with increasing of fluence. This result means that $\mathrm{C}$ atoms in surface of hydrogenated Si-DLC film decreased by exposure an atomic oxygen. Fig 4 shows that the flucence dependence of the $\mathrm{O} 1 s$ spectra measured by XPS of hydrogenated Si-DLC films. As shown in Fig. 4 the $\mathrm{O} 1 \mathrm{~s}$ peak is observable in the spectrum of a) 0 atoms $\mathrm{cm}^{-2}$. It is due to natural oxidation in hydrogenated Si-DLC film surface. The peak at 531 $\mathrm{eV}$ increased with fluence. This result means that $\mathrm{O}$ atoms in surface of hydrogenated Si-DLC film increased by exposure an atomic oxygen. Fig 5 shows that the flucence dependence of the $\mathrm{Si} 2 s$ spectra measured by XPS of hydrogenated Si-DLC films. As shown in Fig. 5 the peak at $155 \mathrm{eV}$ increased with fluence. On the other hand, the peak at $153 \mathrm{eV}$ decreased. Fig 6 shows that the fluence dependence of the Si2 spectra measured by XPS of hydrogenated Si-DLC films. As shown in Fig. 6 the peak at $103.6 \mathrm{eV}$ derived from $\mathrm{SiO}_{2}$ increased with fluence. On the other hand, the peak at $100.3 \mathrm{eV}$ derived from $\mathrm{SiC}$ decreased ${ }^{33)}$. In this result, $\mathrm{Si}$ atoms of hydrogenated Si-DLC film surface are changed to Si-O binding from $\mathrm{Si}-\mathrm{C}$ binding by exposure atomic oxygen. The results of elementary analysis are listed in Table I. These amounts are calculated by relative sensibility coefficient method. The amounts of $\mathrm{Si}$ and $\mathrm{O}$ atom in the surface of the hydrogenated Si-DLC films increased with the fluence of the atomic oxygen beam. However, that of $\mathrm{C}$ atoms decreased. The amounts of $\mathrm{C}$ decreased due to desorption of $\mathrm{C}$ atoms from film surface. On the other hand, $\mathrm{Si}$ amounts increased due to remaining $\mathrm{Si}$ atoms on the film surface.

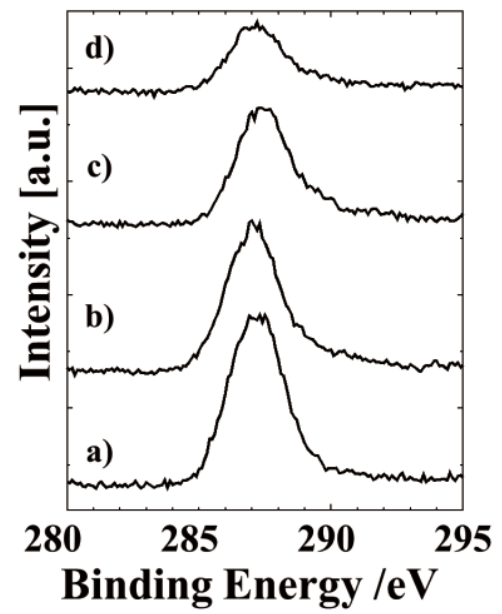

Fig. 3 Fluence dependence of the C $1 s$ XPS spectra of hydrogenated Si-DLC films. Fluence of atomic oxygen beam is (a) 0 atoms $\cdot \mathrm{cm}^{-2}$, (b) $1.2 \times 10^{17}$ atoms $\cdot \mathrm{cm}^{-2}$, (c) $1.2 \times 10^{18}$ atoms $\cdot \mathrm{cm}^{-2}$, (d) $1.2 \times 10^{19}$ atoms $\cdot \mathrm{cm}^{-2}$.

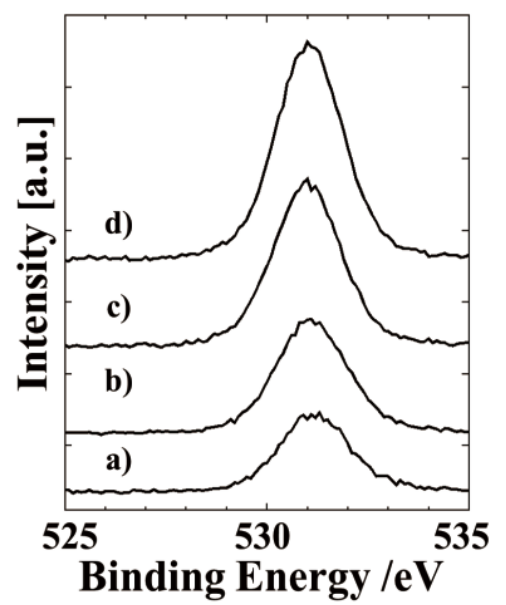

Fig. 4 Fluence dependence of the O $1 s$ XPS spectra of hydrogenated Si-DLC films. Fluence of atomic oxygen beam is (a) 0 atoms $\cdot \mathrm{cm}^{-2}$, (b) $1.2 \times 10^{17}$ atoms $\cdot \mathrm{cm}^{-2}$, (c) $1.2 \times 10^{18}$ atoms $\cdot \mathrm{cm}^{-2}$, (d) $1.2 \times 10^{19}$ atoms $\cdot \mathrm{cm}^{-2}$. 


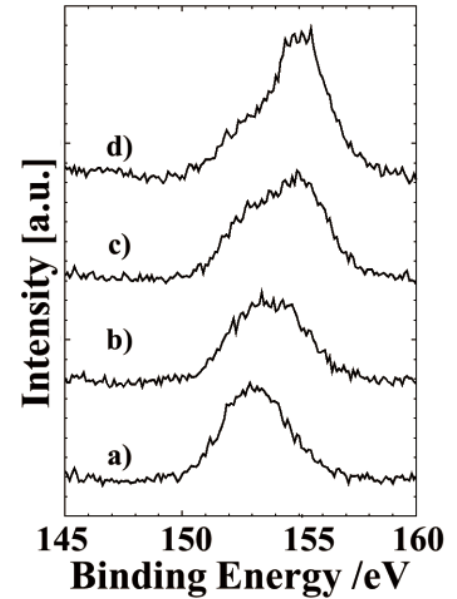

Fig. 5 Fluence dependence of the Si $2 s$ XPS spectra of hydrogenated Si-DLC films. Fluence of atomic oxygen beam is (a) 0 atoms $\cdot \mathrm{cm}^{-2}$, (b) $1.2 \times 10^{17}$ atoms $\cdot \mathrm{cm}^{-2}$, (c) $1.2 \times 10^{18}$ atoms $\cdot \mathrm{cm}^{-2}$, (d) $1.2 \times 10^{19}$ atoms $\cdot \mathrm{cm}^{-2}$.

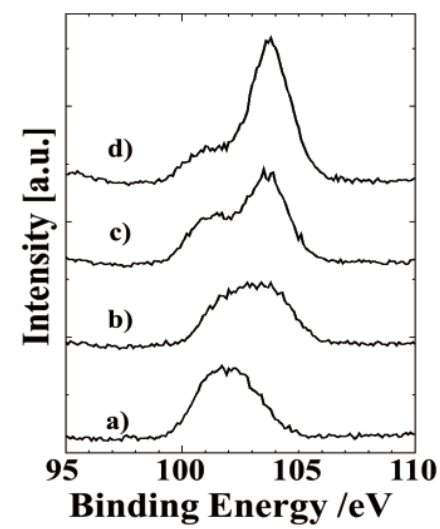

Fig. 6 Fluence dependence of the Si $2 p$ XPS spectra of hydrogenated Si-DLC films. Fluence of atomic oxygen beam is (a) 0 atoms $\cdot \mathrm{cm}^{-2}$, (b) $1.2 \times 10^{17}$ atoms $\cdot \mathrm{cm}^{-2}$, (c) $1.2 \times 10^{18}$ atoms $\cdot \mathrm{cm}^{-2}$, (d) $1.2 \times 10^{19}$ atoms $\cdot \mathrm{cm}^{-2}$.

Table I

Fluence dependence of the Elementary composition of hydrogenated Si-DLC films.

\begin{tabular}{cc|c|c}
\hline Fluence & $\mathrm{C}$ & $\mathrm{O}$ & $\mathrm{Si}$ \\
\cline { 1 - 1 } \cline { 3 - 3 } atoms cm & & at.\% & \\
\hline 0 & & 10 & 37 \\
\hline $1.2 \times 10^{17}$ & 54 & 15 & 40 \\
\hline $1.2 \times 10^{18}$ & 28 & 21 & 51 \\
\hline $1.2 \times 10^{19}$ & 24 & 22 & 55 \\
\hline
\end{tabular}

From the above XPS results, the amounts of $\mathrm{Si}$ and $\mathrm{O}$ atom in the hydrogenated Si-DLC films increased with the fluence of the atomic oxygen beam. However, that of $\mathrm{C}$ atoms decreased monotonically. As results, carbon atoms desorbed from hydrogenated Si-DLC films to form gas species ( $\mathrm{CO}$ and/or $\mathrm{CO}_{2}$ ). Oxygen atoms and silicon atoms generated $\mathrm{Si}$ oxide on surface of hydrogenated Si-DLC film.

Variations in the local structure of the hydrogenated Si-DLC film due to the exposure to an atomic oxygen beam are discussed on the basis of the NEXAFS measurements ${ }^{34)}$. NEXAFS spectroscopy using synchrotron radiation is known to be sensitive to the local structure around the absorber atom. Fig. 7 shows that the fluence dependence of the C $K$-edge NEXAFS spectra of hydrogenated Si-DLC films. The spectrum of a $\mathrm{SiC}$ powder is also shown for reference. A pre-edge resonance at $285.4 \mathrm{eV}$ was originated from $s p^{2}(\mathrm{C}=\mathrm{C})$ sites ${ }^{12.32)}$. The transitions from $\mathrm{C} 1 s$ level to unoccupied $\sigma^{*}$ states were observed in the photon energy region of $290-320 \mathrm{eV}$. Intensity of the sharp peak at $285.4 \mathrm{eV}$ decreased with increasing of fluence of atomic oxygen. On the other hand, intensity of the peak at $289 \mathrm{eV}(\mathrm{C}-\mathrm{O})$ increased. In other words, the $\mathrm{C}-\mathrm{C}$ binding is changed to $\mathrm{C}-\mathrm{O}$ binding by atomic oxygen irradiation. Fig. 8 shows that the fluence dependence of the Si $L$-edge NEXAFS spectra of hydrogenated $\mathrm{Si}-\mathrm{DLC}$ films. The spectra of $\mathrm{SiC}$ powder and $\mathrm{SiO}_{2}$ powder are also shown for reference. In the spectrum of non-irradiated hydrogenated Si-DLC, structual peak was not observed. Intensity of the peak derived from $\mathrm{SiO}_{2}$ increased with fluence of atomic oxygen. After the irradiation of atomic oxygen beyond d) $1.2 \times 10^{19}$ atoms $\cdot \mathrm{cm}^{-2}$, the spectrum changed into a similar shape with the spectrum of $\mathrm{SiO}_{2}$. In other words, the Si-C binding is changed to $\mathrm{Si}-\mathrm{O}$ binding by atomic oxygen irradiation. Fig.9 shows that the Si $K$-edge NEXAFS spectra of hydrogenated Si-DLC films, before and after exposure to an atomic oxygen. The spectra of $\mathrm{SiC}$ powder and $\mathrm{SiO}_{2}$ powder are also shown for reference. The transitions from Si $1 s$ level to unoccupied $\pi^{*}$ states were observed in the photon energy region of $1840 \mathrm{eV}$. With increasing of fluence of atmic oxygen, intensity of the peak at $1848 \mathrm{eV}$ derived from $\mathrm{SiO}_{2}$ increased. Fig. 10 shows that the fluence dependence of the $\mathrm{O} K$-edge NEXAFS spectra of hydrogenated Si-DLC films. The spectrum of a $\mathrm{SiO}_{2}$ powder is also shown for reference. The peak is present in the spectrum of a) 0 atoms $\mathrm{cm}^{-2}$ in the Fig. 10. It is due to natural oxidation in hydrogenated Si-DLC film surface as observed in Fig. 4. With increasing of fluence of atmic oxygen, intensity of the peak $(536 \mathrm{eV})$ derived from $\mathrm{SiO}_{2}$ increased.

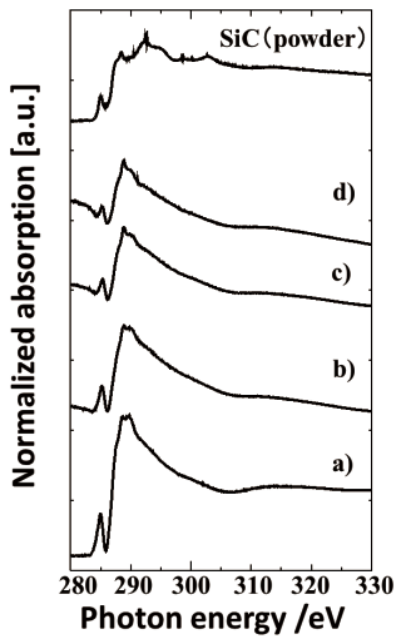

Fig.7 Fluence dependence of the NEXAFS spectra of C $K$-edge of hydrogenated Si-DLC films. Fluence of atomic oxygen beam is (a) 0 atoms $\mathrm{cm}^{-2}$, (b) $1.2 \times 10^{17}$ atoms $\cdot$ $\mathrm{cm}^{-2}$, (c) $1.2 \times 10^{18}$ atoms $\cdot \mathrm{cm}^{-2}$, (d) $1.2 \times 10^{19}$ atoms $\cdot \mathrm{cm}^{-2}$. 


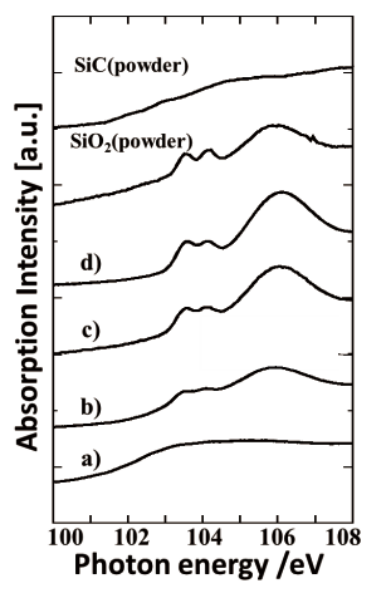

Fig. 8 Fluence dependence of the NEXAFS spectra of Si $L$-edge of hydrogenated Si-DLC films. Fluence of atomic oxygen beam is (a) 0 atoms $\mathrm{cm}^{-2}$, (b) $1.2 \times 10^{17}$ atoms . $\mathrm{cm}^{-2}$, (c) $1.2 \times 10^{18}$ atoms $\cdot \mathrm{cm}^{-2}$, (d) $1.2 \times 10^{19}$ atoms $\cdot \mathrm{cm}^{-2}$.

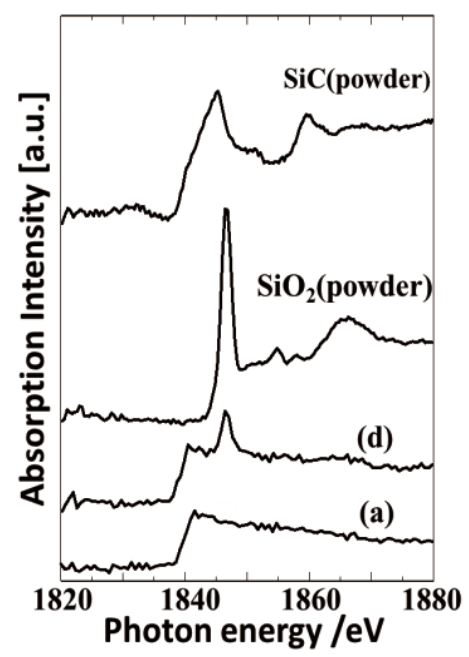

Fig.9 NEXAFS spectra of Si $K$-edge of hydrogenated Si-DLC films, before and after exposure to an atomic oxygen beam. Fluence of atomic oxygen beam is (a) 0 atoms $\cdot \mathrm{cm}^{-2}$, (d) $1.2 \times 10^{19}$ atoms $\cdot \mathrm{cm}^{-2}$.

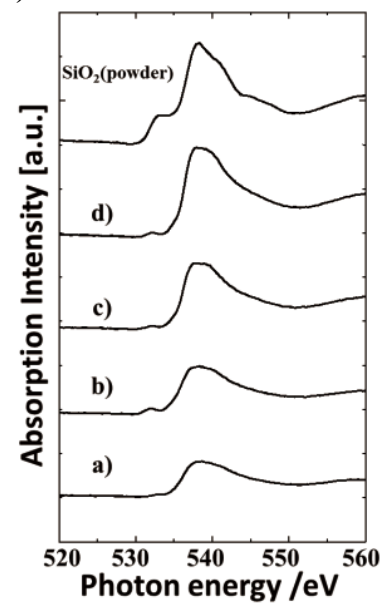

Fig.10 Fluence dependence of the NEXAFS spectra of $\mathrm{O}$ $K$-edge of hydrogenated Si-DLC films. Fluence of atomic oxygen beam is (a) 0 atoms $\cdot \mathrm{cm}^{-2}$, (b) $1.2 \times 10^{17}$ atoms $\cdot$ $\mathrm{cm}^{-2}$, (c) $1.2 \times 10^{18}$ atoms $\cdot \mathrm{cm}^{-2}$, (d) $1.2 \times 10^{19}$ atoms $\cdot \mathrm{cm}^{-2}$.

From the above NEXAFS results, C-C binding is changed to $\mathrm{C}-\mathrm{O}$ binding by atomic oxygen irradiation. $\mathrm{Si}-\mathrm{C}$ binding is changed to $\mathrm{Si}-\mathrm{O}$ binding by atomic oxygen irradiation. As results, carbon atoms desorbed from hydrogenated Si-DLC films to form gas species $\left(\mathrm{CO}\right.$ and/or $\left.\mathrm{CO}_{2}\right)$. Oxygen atoms and silicon atoms generated Si oxide on surface of hydrogenated Si-DLC film. This Si oxide layer is considered to be disturbed the erosion of bulk film against hyperthermal atomic oxygen.

Element distribution in depth direction of hydrogenated Si-DLC films due to the irradiation of atomic oxygen was estimated by the measurement of the GD-OES. Si oxide layer thickness was estimated by GD-OES profile and film thickness. Fig. 11 shows the element analysis of depth direction of $\mathrm{Si}$ at hydrogenated Si-DLC films. The full line and dot-line are shown element analysis of depth direction of 0 atoms $\mathrm{cm}^{-2}$ and $5.5 \times 10^{19}$ atoms $\cdot \mathrm{cm}^{-2}$, respectively. In this figure, the amount of $\mathrm{Si}$ increased in the region of $5 \mathrm{~nm}$ on the film from surface by irradiation of atomic oxygen. Fig. 12 shows the element analysis of depth direction of $\mathrm{O}$ at hydrogenated Si-DLC films. The amount of $\mathrm{O}$ also increased in the region of $5 \mathrm{~nm}$ on the film from surface by irradiation of atomic oxygen. In these results, silicon oxide of formed on the film surface is about $5 \mathrm{~nm}$.

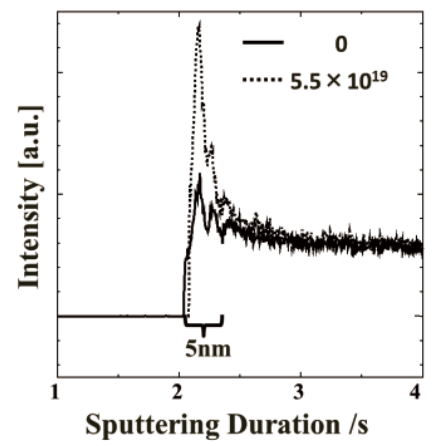

Fig. 11 Element analysis of depth direction of $\mathrm{Si}$ at hydrogenated Si-DLC films. Solid line and broken line is shown atomic oxygen fluence of 0 atoms $/ \mathrm{cm}^{2}$ and $5.5 \times 10^{19}$ atoms $\cdot \mathrm{cm}^{-2}$, respectively.

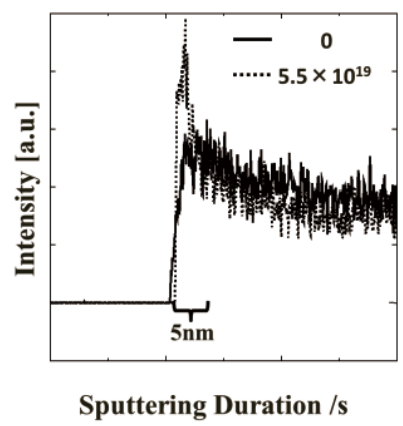

Fig. 12 Element analysis of depth direction of $\mathrm{O}$ at hydrogenated Si-DLC films. Solid line and broken line is shown atomic oxygen fluence of 0 atoms $\cdot \mathrm{cm}^{-2}$ and $5.5 \times 10^{19}$ atoms $\cdot \mathrm{cm}^{-2}$, respectively. 


\section{Conclusion}

We investigated the etching resistance, composition change and resistance mechanism of hyperthermal atomic oxygen beam irradiation on Si-doped hydrogenated Diamond-Like Carbon (hydrogenated Si-DLC) films for the purpose of use as a solid lubrication material in space. It was found that hydrogenated Si-DLC films have resistance to etching by hyperthermal atomic oxygen irradiation since film thickness was constant after exposure to an atomic oxygen. Hydrogenated Si-DLC films, which were exposed to atomic oxygen beam are expected to keep low friction properties in a vacuum, because the hydrogen content in the hydrogenated Si-DLC film after irradiation was $40 \%$ or more. Furthermore, we found the following mechanism for resistance to hyperthermal atomic oxygen irradiation by the measurement of X-ray photoelectron spectroscopy (XPS) and near edge X-ray absorption fine structure (NEXAFS). The carbon atoms on the hydrogenated Si-DLC surface desorbed as the $\mathrm{CO}$ and/or $\mathrm{CO}_{2}$, but silicon atoms were remained on the hydrogenated $\mathrm{Si}$-DLC surface as the $\mathrm{SiOx}$. It was interpreted that this SiOx layer on the surface disturbed the erosion of bulk film against atomic oxygen.

The thickness of Si oxide, formed on the surface by exposed atomic oxygen, was about $5 \mathrm{~nm}$ from the measurement of the GD-OES. In this reason, it is seem that $\mathrm{SiOx}$ layer is not involved in the friction properties because solid lubrication material performs while scraping the surface. We are planning to examine friction coefficient of hydrogenated Si-DLC film, after exposure an atomic oxygen beam. From this study, hydrogenated Si-DLC films are concluded to be expectable as a lubrication material in LEO, because hydrogenated Si-DLC has high resistance to atomic oxygen irradiation.

\section{Acknowledgments}

We thank staff for use of 5SDH-2 of the Accelerator and Particle Beam Experimental Facility, Faculty of Maritime Sciences, Kobe University in the ERDA/RBS measurements. This work was supported in part by Hyogo Science and Technology Association.

\section{Reference}

[1] B. A. Banks, K. K. de Groh, S. K. Miller, NASA/TM-2004-213400 (2004) E-14905.

[2] J. H. Han, C. G. Kim, Composite Structures, 72 (2006) 218-26.

[3] E. R. Crutcher, L. S. Nishimura, K. J. Warner, and W. W. Wascher, LDEF-69 Months in Space: First Post-Retrieval Symposium, June 2-8, NASA CP 3134 , (1991) 861-74.

[4] S. W. Samwel, Space Res. J., 7(1) (2014) 1-13.

[5] J. Robertson, Surf. Coat. Technol., 50 (1992)

185-203.

[6] S. Aisenberg, S. Chabot, Carbon 10, (1972) 356.

[7] J. Robertson, Materials and Engineering, R 37 (2002) 129-281.

[8] K. Bewilogua, D. Hofmann, Surf. Coat. Technol., 242 (2014) 214-225.

[9] J. K. Luo, Y. Q. Fu, H. R. Le, J. A. Williams, S. M.
Spearing, W. I. Milne, J. Micromech. Microeng., 17 (2007) S147-S163.

[10] B. Podogornik, J. Vizintin, Surf. Coat. Technol., 200 (2005) 1982-89.

[11] C. Donnet, M. Belin, J. C. Auge, J. M. Martin, A. Grill, V. Patel, Surf. Coat. Technol., 68/69 (1994) 626-31. [12] M. Tagawa, K. Kumiko, A. Kitamura, K. Matsumoto, A. Yoshigoe, Y. Traoka, K. Kanda, M. Niibe, Appl. Surf. Sci., 256 (2010) 7678-83.

[13] J. H. Ouyang, S. Sasaki, Surf. Coat. Technol., 195 (2005) 234-44.

[14] L. Cui, L. Guoqing, C. Wenwu, M. Zongxin, Z. Chengwu, W. Liang, Thin Solid Films, 475(2005) 279-82.

[15] M. Lubwama, B. Corcoran, K.A. McDonnell, D. Dowling, J.B. Kirabira, A. Sebbit, K. Sayers, Surf. Coat. Technol., 239 (2014) 84-94.

[16] A. A. Voevodin, C. Rebholz, A. Matthews, Tribol. Trans., 38 (1995) 829-36.

[17] S. R. P. Silva, J. Robertson, G. A. J. Amaratunga, J.Appl. Phys., 81 (1997) 2626-34.

[18] K. Baba, R. Hatada, Surf. Coat. Technol., 169 (2003) 287-90.

[19] A. Amanov, T. Watave, R. Tsuboi, S. Sakaki, Suf. Coat. Technol., 232 (2013) 549-60.

[20] K. Oguri, T. Arai, Surf. Coat. Technol., 47 (1991) 710-21.

[21] M. Kim, K. Lee, K. Eun, Surf. Coat. Technol., 112 (1999) 204-09.

[22] A. Varma, V. Palshin, E. I. Meletis, Surf. Coat. Technol., 148 (2001) 305-14.

[23] T. Iseki, H. Mori, H. Hasegawa, H. Tachikawa, K. Nakanishi, Diamond \& Related Materials, 15 (2006) 1004-10.

[24] D. Hofmann, S. Kunkel, K. Bewilogua, R. Wittorf, Surf. Coat. Technol., 215 (2013) 357-63.

[25] T. Nakahigashi, Y. Tanaka, K. Miyake, H. Oohara, Triol. Int., 37 (2004) 907-12.

[26] M. Tagawa, K. Yokota, N. Ohmae, H. Kinoshita, and M. Umeno, Jpn. J. Appl. Phys., 40 (2001) 6152-56.

[27] K. Yokota, M. Tagawa, N. Ohmae , J. Spacecraft Rockets, 40 (2003) 143-44.

[28] D. K. Avasthi, and W. Assmann, vol.80, NO.12 (2001) 1532-41.

[29] M. Niibe, M. Mukai, S. Miyamoto, Y. Shoji, S. Hashimoto, A. Ando, T. Tanaka, M. Miyai, H. Kitamura, AIP Conf. Proc., 705 (2004) 576-79.

[30] M. Niibe, M. Mukai, H. Kimura, Y. Shoji, AIP Conf. Proc., 705 (2004) 243-246.

[31] A. Saikubo, N. Yamada, K. Kanda, S. Matsui, T. Suzuki, K. Niihara, H. Saitoh, Diamond \& Related Materials, 17 (2008) 1743-45.

[32] A. Saikubo, K. Kanda, M. Niibe, S. Matsui, New Diamond Front. Carbon Technol., 16 (2006) 235-44.

[33] Y. Hijikata, H. Yaguchi, M. Yoshikawa, S. Yoshida, Applied Surface Science, 184 (2001) 161-66.

[34] K. Kanda, M. Niibe, A. Wada, H. Ito, T. Suzuki, T. Ohana, N. Ohtake, and H. Saitoh, Jpn. J. Appl. Phys., 52 (2013) 095504.

(Received January 31, 2015; Accepted May 16, 2015) 